\title{
Non-initial overlap bulk silicon comb-drive actuator with low-voltage and large-displacement
}

\author{
Chunhua Cai ${ }^{1}$, Di Hua ${ }^{1}$ and Ming Qin ${ }^{2}$ \\ ${ }^{1}$ College of internet of things engineering, Hohai University, 213022 Changzhou, China \\ ${ }^{2}$ Key Laboratory of MEMS of the Ministry of Education, Southeast University, 210096 Nanjing, China
}

\begin{abstract}
Two different kinds of bulk-Si comb-drive actuators are designed in this work: initial overlap comb-drive actuator and non-initial overlap comb-drive actuator. They are fabricated by a simple post-CMOS bulk micromachining process. A cascade folded beam is designed to achieve large displacement at low driving voltages. And non-initial overlap and unequal wide comb fingers are used to reduce the side instability and improve the pull-in voltage. The measurement results show that the non-initial overlap comb-drive actuator improves the pull-in voltage by $73.3 \%$ than the initial overlap comb-drive actuator, and the maximum displacement of the non-initial overlap actuator is larger by $78.9 \%$ than that of the initial overlap actuator.
\end{abstract}

\section{Introduction}

Comb-drive actuators based on the principle of electrostatic forces have become a typical component in MEMS devices due to its low power consumption, high speed and good compatibility with microfabrication process [1]. Since the first time developed by Tang et al. in 1989 [2], comb-drive actuators have be used in a variety of applications including tweezer [3], optical switch [4], micro-tribotester [5] and Micro XY-stage [6$8]$, etc.Generally, comb-drive actuators usually need a high drive voltage to achieve a large displacement. In addition, the desired displacement of the comb-drive actuators is constrained by the side instability. In order to overcome this drawback, some effective approaches have been developed for a large displacement by alleviating side instability, such as optimal suspension designs [9-11], linearly engaging comb fingers [12-13], and appropriate first and last comb fingers [14]. However, there is scarcely comb-drive actuator that can achieve $50 \mu \mathrm{m}$ within $25 \mathrm{~V}$ driving voltages [9-17].

In this work, the non-initial overlap and unequal wide comb fingers are utilized to reduce the side instability and improve the pull-in voltage. A cascade folded beam is used to achieve to achieve the displacement of more than $50 \mu \mathrm{m}$ at $24 \mathrm{~V}$ driving voltages within a relatively small size. The whole size of the comb-drive actuator is $1.14 \mathrm{~mm}^{2}$. In addition, the comb-drive actuators are fabricated using a post-CMOS bulk micromachining process.

Furthermore, the fabrication tolerances together with their effects on the performances of the comb-drive actuator are also discussed.

\section{The post-CMOS process}

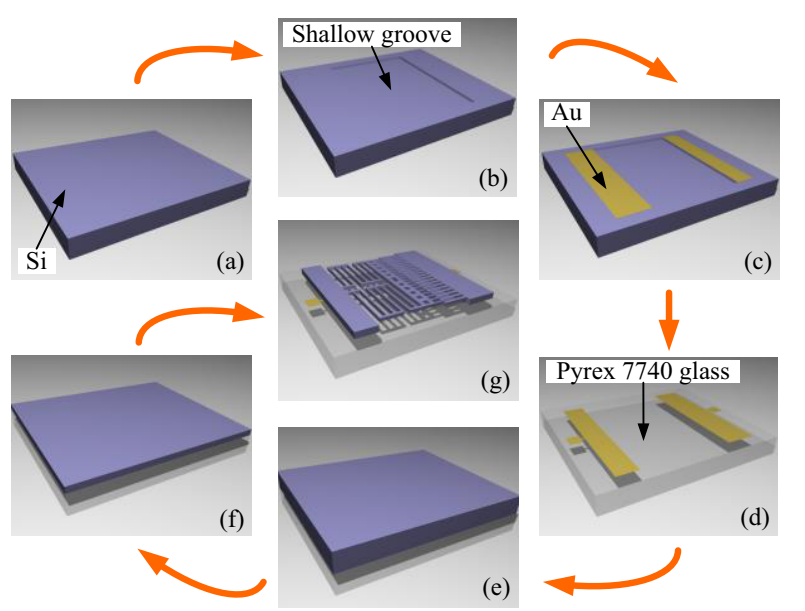

Figure 1. Fabrication process of the comb-drive actuator based on post-CMOS technology.

This research demonstrates a bulk silicon post-CMOS MEMS process based on the $\mathrm{Au}-\mathrm{Au}$ bonding technology to fabricate the electrostatic type comb-drive actuators. In Figure 1 (b), the $4 \mu \mathrm{m}$ shallow grooves with precise geometry are fabricated on the silicon substrate (Figure 1 (a)) by RIE (reactive ion etch) process, leaving a small clearance where the movable comb fingers of the combdrive actuators can extend on the glass chip. As shown in Figure 1 (c) and Figure 1 (d), $500 \AA / 1000 \AA \mathrm{Ti} / \mathrm{Au}$ is sputtered onto the Pyrex7740 glass wafer and the silicon substrate. As indicated in Figure 1 (e), the silicon substrate is flip-chipped onto the Pyrex7740 glass wafer 
by using the $\mathrm{Au}-\mathrm{Au}$ thermal bonding process. The $\mathrm{Au}-$ $\mathrm{Au}$ bonding technique is practicable, reliable, and capable of providing pads for electrical interconnection and hermetic packing of MEMS devices [18].

Then the Si wafer is thinned to about $34 \mu \mathrm{m}$ from the backside by the CMP (chemical mechanical planarization) process at the temperature of around $30{ }^{\circ} \mathrm{C}$ with the alkaline $\mathrm{SiO}_{2}$ slurry (Figure 1 (f)). At last, the Bulk-Si micro-structures are released through the ICP (inductively coupled plasma) process by the ionized gas of $\mathrm{C}_{4} \mathrm{~F}_{8}$ (Figure $1(\mathrm{~g})$ ).

\section{Design of the bulk silicon comb-drive actuators}
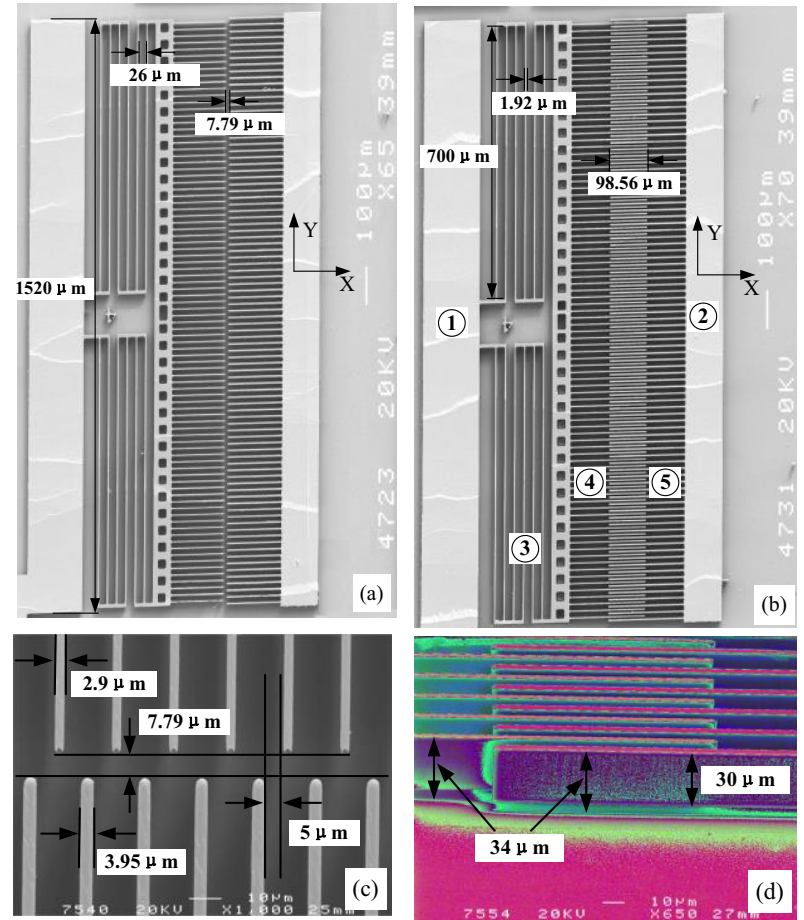

Figure 2. SEM photos of the comb-drive actuators: (a) Noninitial overlap comb-drive actuator, (b) Initial overlap combdrive actuator, (c) Non-initial overlap comb fingers, (d) Profiles of the initial overlap comb fingers. (1) (2)-Input pads of driving voltages and output pads of capacitance, (3)- Folded beams, (4)Movable comb fingers, (5)-Fixed comb fingers.

With the development of the comb-drive actuators techniques, bigger stroke, higher speed and higher accuracy are required in various applications. However, some obstacles have been present accordingly, such as side instability, high applied voltage and large device footprint. To overcome these shortcomings, a novel noninitial overlap bulk silicon comb-drive actuator is designed and fabricated in this work to achieve a large displacement at a low driving voltage within a relatively small size, as shown in Figure 2 (a). As a comparison, an initial overlap comb-drive actuator and a non-initial overlap comb-drive actuator are described as shown in Figure 2 (b). The sizes of the two actuators are the same except overlap segments.

As shown in Figure 2(b), the electrostatic force $F_{x}$ generated between the movable comb fingers and the fixed comb fingers along the moving direction ( $\mathrm{x}-$ direction) is given as:

$$
F_{x}=\frac{N \varepsilon_{0} t U^{2}}{g}
$$

where $N$ is the number of movable comb fingers, $\varepsilon_{0}$ is the permittivity constant of air, $t$ is the thickness of the comb-drive fingers, $g$ is the fingers gap spacing between the movable comb finger and the fixed comb finger and $U$ is the applied voltage.

As shown in Figure 2(a), the movable comb fingers and the fixed comb fingers of the comb-drive actuator are not overlap. With a driving voltage $U$ and the comb fingers non overlap, the electrostatic force $F_{x \text {-non-overlap }}$ generated between the movable comb drives and the fixed comb drives along the moving direction ( $\mathrm{x}$ direction) is given as

$$
F_{x-\text { non-overlap }}=\frac{1}{2} \frac{N \varepsilon_{0}\left(w_{m}+w_{f}\right) t U^{2}}{\left(L+L_{1}-x\right)^{2}}
$$

Where $w_{m}$ is the width of movable fingers, $w_{f}$ is the width of fixed comb fingers, $L$ is the length of the comb finger, $L_{l}$ is the length of the non-initial overlap segment, and $x$ is the displacement of the movable comb fingers in the moving direction.

Based on equation (1) and equation (2), the electrostatic force ratio of non-overlap comb fingers and overlap comb fingers can be expressed as:

$$
\frac{F_{x-\text { non-overlap }}}{F_{x}}=\frac{1}{2} \frac{\left(w_{m}+w_{f}\right) g}{\left(L+L_{1}-x\right)^{2}}
$$

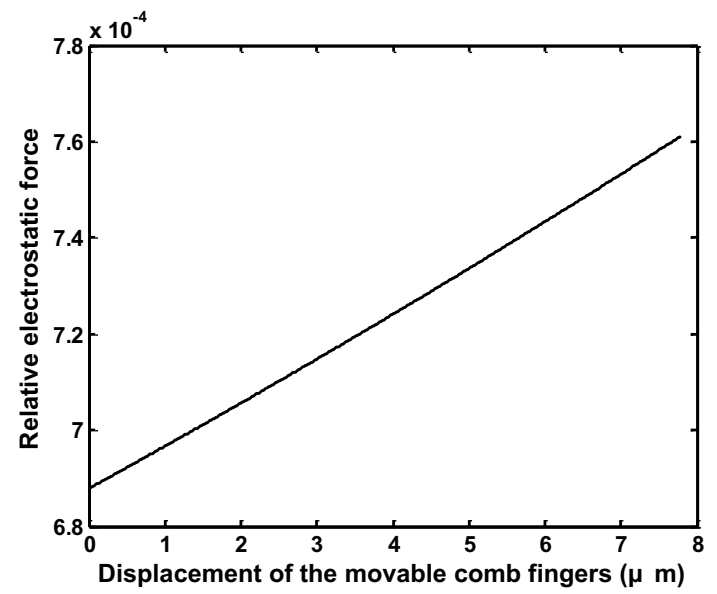

Figure 3. The relative electrostatic force versus the displacement of the movable comb fingers.

Figure 3 shows the electrostatic force ratio as a function of the displacement of the movable comb fingers. Under the conditions of thickness $t=30 \mu \mathrm{m}$, gap between comb fingers $g=5 \mu \mathrm{m}$, width of the movable comb fingers $w_{m}=2.9 \mu \mathrm{m}$, width of the fixed comb fingers $w_{f}=$ $3.95 \mu \mathrm{m}$, length of comb fingers $L=150 \mu \mathrm{m}$, length of the non-initial overlap segment $L_{1}=7.79 \mu \mathrm{m}$. When the displacement of the movable comb fingers increases from 0 to $7.79 \mu \mathrm{m}$, the relative electrostatic force of the combdrive actuator increases from $6.88 * 10^{-4}$ to $7.61 * 10^{-4}$. The curve derives that the displacement change rate of the non-initial overlap actuator with the comb fingers nonoverlap are lower than that of the non-initial overlap actuator with the comb fingers overlap. 


\section{Tolerance of DRIE effects on the performance of the actuators}

Due to the etching tolerance by deep reactive ion etching (DRIE), the measured results are different from the initial design. Generally, wider trenches tend to become more reentrant. Consequently, the fabrication tolerance generated by DRIE affects the electrical and mechanical performance of the comb-drive actuators [19].

\subsection{Tolerance of DRIE effects on the capacitance} of the initial overlap comb-drive actuator

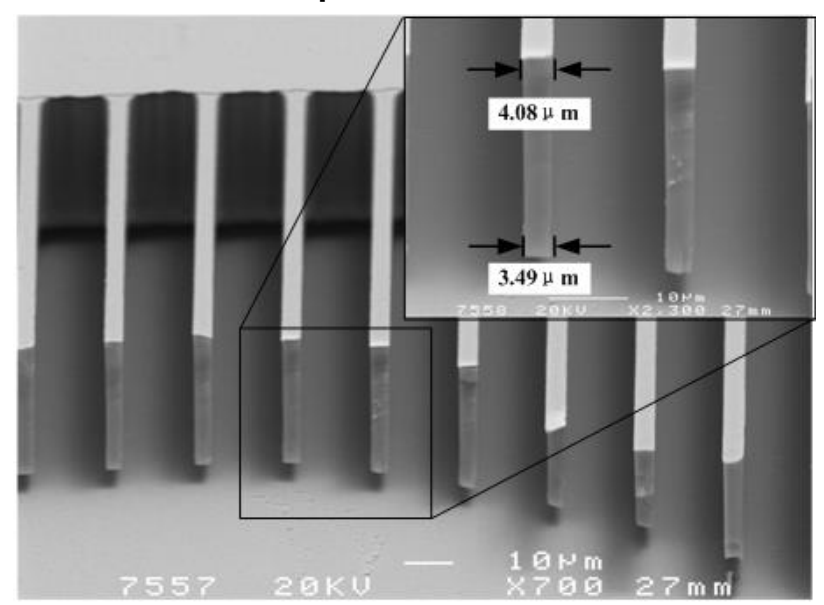

(a)

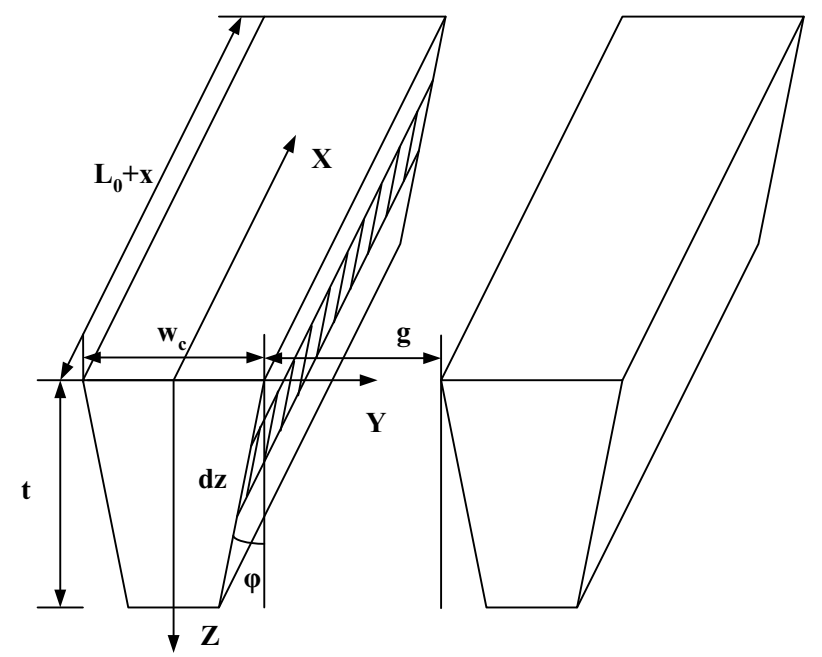

(b)

Figure 4. The fixed comb fingers with negative etching profile: (a) SEM images of the broken fixed comb fingers; (b) Scheme of the comb fingers with negative etching profile.

The profile tolerance of comb fingers and flexure beams caused by the DRIE process were studied [19]. Figure 4 (a) shows the SEM images of the comb fingers with the negative profile which are measured from the intentionally broken pieces of the comb-drive actuators. The thickness of the comb fingers and the cascade folded beams is $30 \mu \mathrm{m}$. The capacitance between the movable comb fingers and the fixed comb fingers, which excludes the fringing capacitance, could be expressed as:

$$
C_{\text {tot-profiles }}=-N \cdot \frac{\varepsilon_{0}\left(L_{0}+x\right)}{\tan \varphi} \cdot \ln \left(1-\frac{t}{g} \cdot 2 \tan \varphi\right)
$$

\subsection{Tolerance of DRIE effects on Stiffness of the Folded Beams}

Due to the etching tolerance by deep reactive ion etching (RIE), the cascade folded beams also have negative profiles. And wider trenches tend to become more reentrant [19]. As a result, the slope angle $\theta$ of the cascade folded beams is around $-1.2^{\circ}$. The negative profiles effect on the stiffness of the folded beams. Consequently, they effect on the performances of the comb-drive actuator.

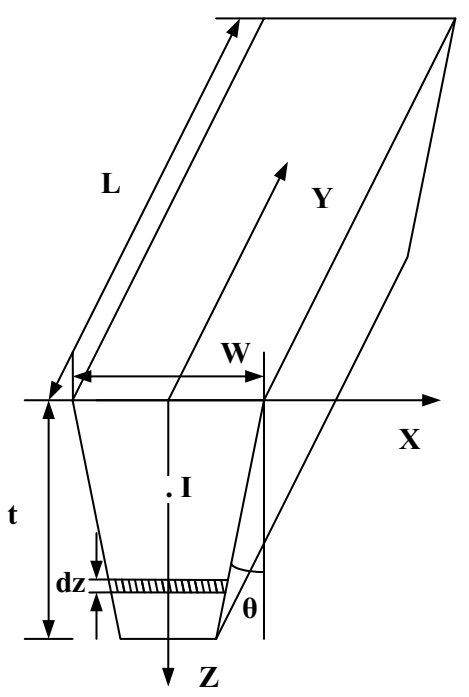

Figure 5. Scheme of the negative etching profiles of the cascade folded beam with fabrication tolerance caused by the DRIE process.

Figure 5 shows a scheme of the folded beam with the negative slope profile. Assuming that the width of the flexure is $W$ and slope angle is $\theta$. The width of the folded beam becomes the function of the depth as expressed as:

$$
w(z)=W+2 z \cdot \tan \theta
$$

The inertial momentum $I_{x-\text { slope }}$ and the stiffness in the x-direction of the folded beams $K_{x}$ is calculated as:

$$
\begin{gathered}
I_{\mathrm{x}-\text { slope }}=\int_{0}^{t} \frac{(W+2 z \cdot \tan \theta)^{3}}{12} d z \\
=\frac{(2 t \cdot \tan \theta+W)^{4}-W^{4}}{96 \cdot \tan \theta} \\
K_{x-\text { slope }}=\frac{12 E I}{L^{3}} \cdot \frac{4}{3}=E \cdot \frac{(2 t \cdot \tan \theta+W)^{4}-W^{4}}{6 \cdot \tan \theta \cdot L^{3}}
\end{gathered}
$$

\subsection{Tolerance of DRIE effects on deflection of the comb-drive actuator}

With a driving voltage $U$, the electrostatic force $F_{x}$ generated between the movable comb fingers and the fixed comb fingers along the moving direction ( $\mathrm{x}$ direction) is given as

$$
F_{x-\text { profiles }}=\frac{\partial}{\partial \mathrm{x}}\left(\frac{1}{2} C_{\text {tot-profiles }} U^{2}\right)
$$

For the overlap comb fingers structure with consideration of edge field, the electrostatic force $F_{x}$ 
along the moving direction( $\mathrm{x}$-direction) and the displacement can be expressed as:

$$
F_{x-\text { profiles }}=-\frac{N}{2} \cdot \frac{\varepsilon_{0}}{\tan \varphi} \cdot \ln \left(1-\frac{t}{g} \cdot 2 \tan \varphi\right) \cdot U^{2}
$$

Based on equation (7) and (9), the displacement of the comb-drive actuator with negative etching profiles can be calculated as:

$$
x_{\text {profiles }}=\frac{-\frac{N}{2} \cdot \frac{\varepsilon_{0}}{\tan \varphi} \cdot \ln \left(1-\frac{t}{g} \cdot 2 \tan \varphi\right) \cdot U^{2}}{E \cdot \frac{(2 t \cdot \tan \theta+W)^{4}-W^{4}}{6 \cdot \tan \theta \cdot L^{3}}}
$$

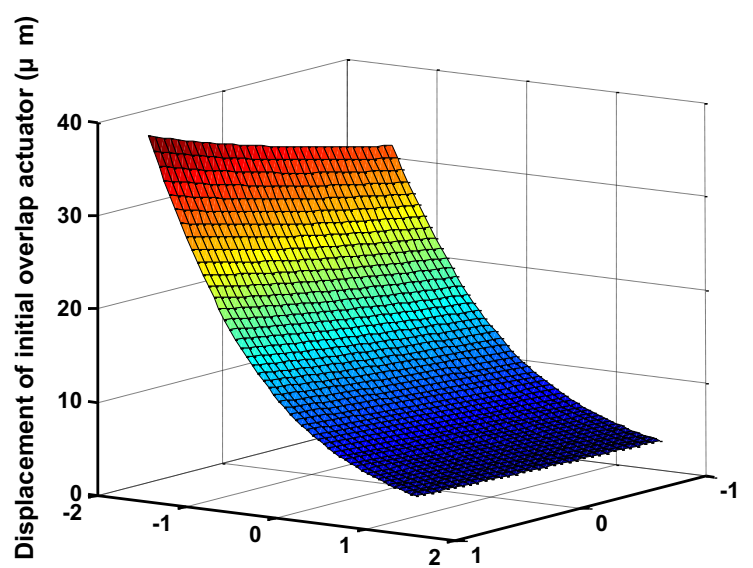

Folded beams slop angle (deg.) Comb fingers slop angle (deg.)

Figure 6. Displacement of comb-drive actuator versus slope angle of folded beams and comb fingers.

Figure 6 shows that the displacement of the actuator increases rapidly when the slope angle of the folded beams $\theta$ decreases. On the contrary, the displacement of the actuator increases slowly when the slope angle of the comb fingers $\varphi$ increases. Excluding the fringing capacitance of the actuator, the displacement of the comb-drive actuator is $24.612 \mu \mathrm{m}$ with the slope angle of comb fingers $\varphi=-0.5634^{\circ}$, the slope angle of the fold beam $\theta=-1.2^{\circ}$ and the driving voltage $U=15 \mathrm{~V}$.

\section{Measurements}

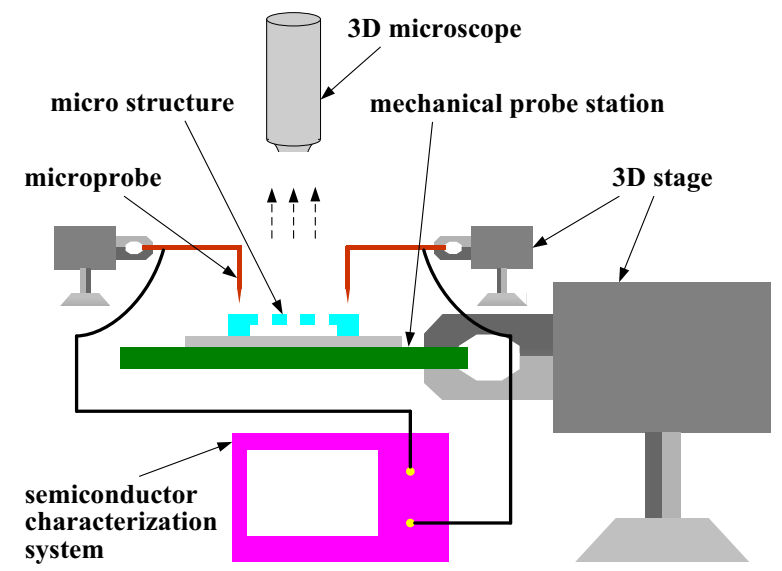

Figure 7. Schematic of the test system.

The comb-drive actuator is measured by the HiROX HI-SCOPE Advanced KH-3000 3D microscopy and
KEITHLEY 4200-SCS semiconductor characterization system with the applied voltage in the range from $0 \mathrm{~V}$ to $15 \mathrm{~V}$ for the initial overlap comb-drive actuator and $0 \mathrm{~V}$ to $24 \mathrm{~V}$ for the non-initial overlap comb-drive actuator.

As shown in Figure 8, the displacement change rate of the initial overlap comb-drive actuator is $0.125 \mu \mathrm{m} / \mathrm{V}^{2}$. The initial overlap actuator is pulled in at $15 \mathrm{~V}$ driving voltages. The maximum displacement of the initial overlap actuator is $28.5 \mu \mathrm{m}$. The measured displacement change rate of the initial overlap actuator is larger by $186.7 \%$ than the initial design value. That is the reason that the folded beams have negative etching profiles. The negative etching profiles decrease the stiffness of the folded beams by $62.35 \%$. Consequently, that will increase the deflection of the comb-drive actuator by $165.5 \%$. Consistency between the experimental results of the initial overlap comb-drive actuator and the modified designed curve that considered the fabrication tolerance is observed. For the fringing capacitance between the movable comb fingers and the fixed comb fingers of the initial overlap comb-drive actuator is excluded, the electrostatic force of the initial overlap comb-drive actuator decreases. As a result, the measured displacement of the initial overlap comb-drive actuator is larger than the modified displacement $x_{\text {profiles }}$ of the actuator that considered the fabrication tolerance.

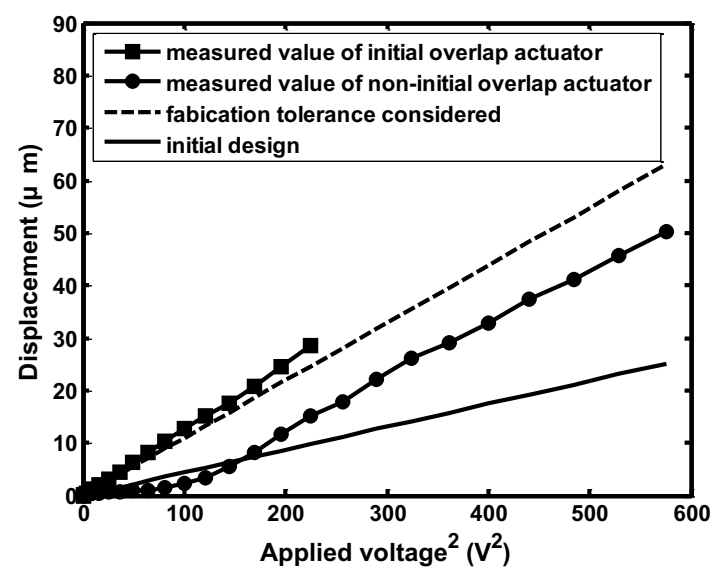

Figure 8. Measured results of displacement of the two kinds of comb-drive actuators.

The displacement change rate of the non-initial overlap comb-drive actuator is $0.03 \mu \mathrm{m} / \mathrm{V}^{2}$ and the comb fingers of the non-initial overlap actuator are non-overlap with the driving voltage in the range from $0 \mathrm{~V}$ to $14 \mathrm{~V}$. And the displacement change rate of the non-initial overlap combdrive actuator is $0.114 \mu \mathrm{m} / \mathrm{V}^{2}$ and the comb fingers of the non-initial overlap comb-drive actuator are overlap with the driving voltage in the range from $15 \mathrm{~V}$ to $24 \mathrm{~V}$. The pull-in voltage of the non-initial overlap actuator is $24 \mathrm{~V}$. And the maximum displacement of the non-initial overlap actuator is $50.127 \mu \mathrm{m}$.

\section{Conclusions}

Two kinds of bulk silicon comb-drive actuators with low voltage and large displacement has been designed and fabricated by a novel and simple bulk 
micromachining post-CMOS process. A cascade folded beam is designed to achieve large displacement at low driving voltages. And non-initial overlap and unequal wide comb fingers are used to reduce the side instability and improve the pull-in voltage. The measurement results show that the non-initial overlap comb-drive actuator improves the pull-in voltage by $73.3 \%$ than the initial overlap comb-drive actuator, and the maximum displacement of the non-initial overlap actuator is larger by $78.9 \%$ than that of the initial overlap actuator. With the optimized cascade folded beams and the unequal wide and non-initial overlap comb fingers, the displacement of the non-initial overlap actuator can reach $50.127 \mu \mathrm{m}$ at $24 \mathrm{~V}$ driving voltages. The fabrication tolerances together with their effects on the performances of the comb-drive actuator are also discussed. The experimental results of the comb-drive actuator are in good agreement with the theoretical predictions.

\section{Acknowledgment}

This work was supported by the Fundamental Research Funds for the Central Universities (2014B02014) and the National High Technology Research and Development Program of China (2013AA041106).

\section{References}

1. J. Li, Q. X. Zhang, and A. Q. Liu, "Advanced fiber optical switches using deep RIE (DRIE) fabrication," Sensors and Actuators A(physical), vol. 102, no. 3, pp.286-295, Jan. 2003.

2. W. C. Tang, T. C. H. Nguyen, and R. T. Howe, "Laterally driven polysilicon resonant microstructures," Sensors and Actuators A(physical), vol. 20, no. 1-2, pp.25-32, Nov. 1989.

3. X. Liu, J. Tong and Y. Sun, "A millimeter-sized nanomanipulator with sub-nanometer positioning resolution and large force output," Smart Mater. Struct, vol. 16, no. 5, pp.1742-1750, Oct. 2007.

4. K. Wang, M. Sinclair, G. K. Starkweather, and K. F. Böhringer, "An Electrostatic Zigzag Transmissive Microoptical Switch for MEMS Displays," Journal of Microelectromechanical Systems, vol. 16, no. 1, pp.140-154, Feb. 2007.

5. Z. Guo, Y. Meng, H. Wu, C. Su, and S. Wenb, "Measurement of static and dynamic friction coefficients of sidewalls of bulk-microfabricated MEMS devices with an on-chip micro-tribotester," Sensors and Actuators A(physical), vol. 135, no. 2, pp.863-869, Apr. 2007.

6. T. Harness, R. R. A. Syms, "Characteristic modes of electrostatic comb-drive X-Y microactuators," Journal of Micromechanics and Microengineering, vol. 10, no. 1, pp.7-14, Mar. 2000.

7. C. H. Kim, H. M. Jeong, J. U. Jeon, and Y. K. Kim, "Silicon Micro XY-Stage With a Large Area Shuttle and No-Etching Holes for SPM-Based Data Storage," Journal of Microelectromechanical Systems, vol. 12, no. 4,pp.470-478, Aug. 2003.
8. L. Gu, X. Li, H. Bao, B. Liu, Y. Wang, M. Liu, Z. Yang and B. Cheng, "Single-wafer-processed nanopositioning XY-stages with trench-sidewall micromachining technology," Journal of Micromechanics and Microengineering, vol. 16, no. 7, pp.1349-1357, Jul. 2006.

9. G. Zhou, and P. Dowd, "Tilted folded-beam suspension for extending the stable travel range of comb-drive actuators," Journal of Micromechanics and Microengineering, vol. 13, no. 2, pp.178-183, Mar. 2003.

10. S. Krylov, and Y. Bernstein, "Large displacement parallel plate electrostatic actuator with saturation type characteristic," Sensors and Actuators A(physical), vol. 130-131, no. 14, pp.497-512, Aug. 2006.

11. M. A. Erismis, H. P. Neves, R. Puers, and C. V. Hoof, "A low-voltage large-displacement large-force inchworm actuator," Journal of Microelectromechanical Systems, vol. 17, no. 6, pp.1294-1301, Dec. 2008.

12. A. Ming, T. Li, P. Zhou, and Y. Wang, "An Electrostatic MEMS Actuator with Large Displacement Under Low Driving Voltage,” Journal of semiconductors, vo1. 29, no. 9, pp.1703-1707, Sep. 2008

13. J. D. Grade, H. Jerman, and T. W. Kenny, "Design of Large Deflection Electrostatic Actuators," Journal of Microelectromechanical Systems, vol. 12, no. 3, pp.335-343, Jun. 2003.

14. Y. C. Chen, I. C. M. Chang, R. Chen, and M. T. K. Houc, "On the side instability of comb-fingers in MEMS electrostatic devices," Sensors and Actuators A(physical), vol. 148, no. 1, pp.201-210, Nov. 2008.

15. A. E. Franke, J. M. Heck, T.-J. King, and R. T. Howe, "Polycrystalline silicon-germanium films for integrated microsystems," Journal of Microelectromechanical Systems, vol. 12, no. 2, pp.160-171, Apr. 2003.

16. J. C. Chiou and C. F. Kuo, "Development of Vertical Electrostatic Comb-Drive Actuator Using Magnified Cascade Configuration," Japanese Journal of Applied Physics, vol. 46, no. 10A, pp.6546-6549, Oct. 2007.

17. Y. Gerson, S. Krylov, B. Ilic, and D. Schreiber, "Large displacement low voltage multistable micro actuator," Proceedings of the IEEE 21st International Conference on Micro Electro Mechanical Systems, pp.463-466, Jan. 2008.

18. R. F. Wolffenbuttel, and K. D. Wise, "Lowtemperature silicon wafer-to-wafer bonding using gold at eutectic temperature," Sensors and Actuators A(physical), vol. 43, no. 1-3, pp.223229, May. 1994.

19. E. Jing, B. Xiong, and Y. L. Wang, "Low temperature Au-Si wafer bonding," Journal of Micromechanics and Microengineering, vol. 20, no. 9, 095014(6pp), Sep. 2010. 